\begin{tabular}{l} 
Final Report Certification \\
for \\
CRADA Number 0050 \\
\hline
\end{tabular}

Between

UT-Battelle, LLC

and

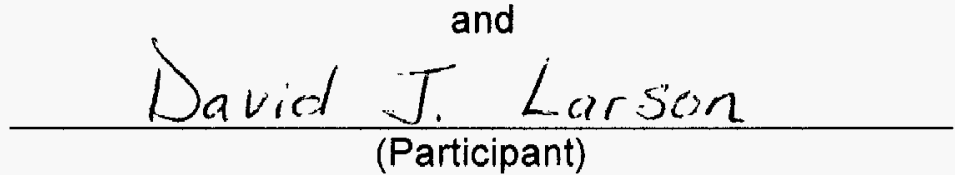

Instructions:

Mark the appropriate statement in $1 \mathrm{a}$ or $1 \mathrm{~b}$ below with an "IX." Refer to the articles in the CRADA terms and conditions governing the identification and marking of Protected CRADA Information (PCl).

If no $\mathrm{PCl}$ is identified, the report will be distributed without restriction. If $\mathrm{PCl}$ is identified, the report distribution will be limited in accordance with the CRADA terms and conditions governing release of data. In all cases items 2 and 3 must be true. That is, the report cannot contain Proprietary Information and a disclosure must be filed prior to release of the report.

This certification may either be made by using this form or may be made on company letterhead if the Participant desires. A faxed copy of this completed form is acceptable.

The following certification is made for the subject final report:

1. (a) The final report contains information that qualifies as "Protected CRADA Information" (PCl). The PCl legend is printed on the report cover, and the $\mathrm{PCl}$ is clearly identified.

OR

(b) $\square$ The final report does not contain "Protected CRADA Information." The "Approved for Public Release" legend is printed on the report cover.

2. The final report does not contain Proprietary Information.

3. By the signature below, the Participant has no objection to the public distribution of the final report due to patentable information.
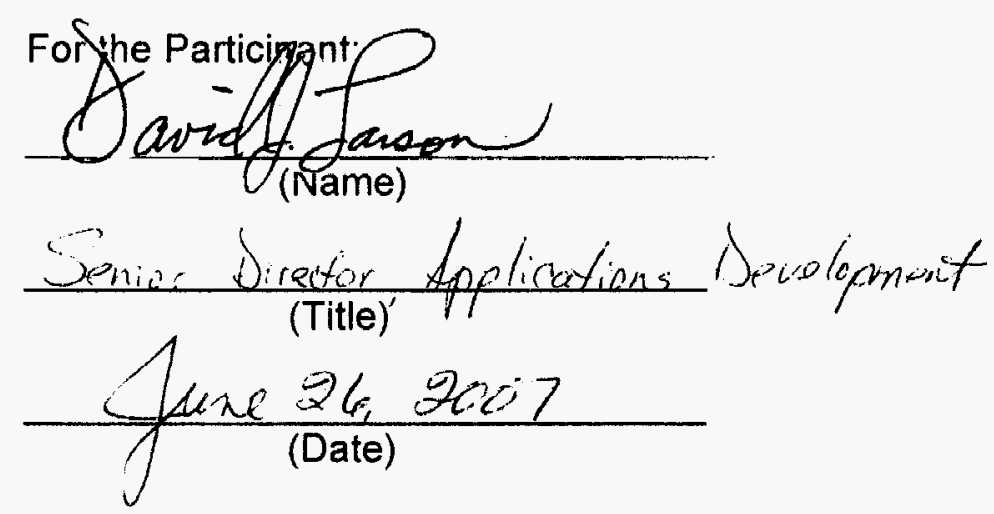


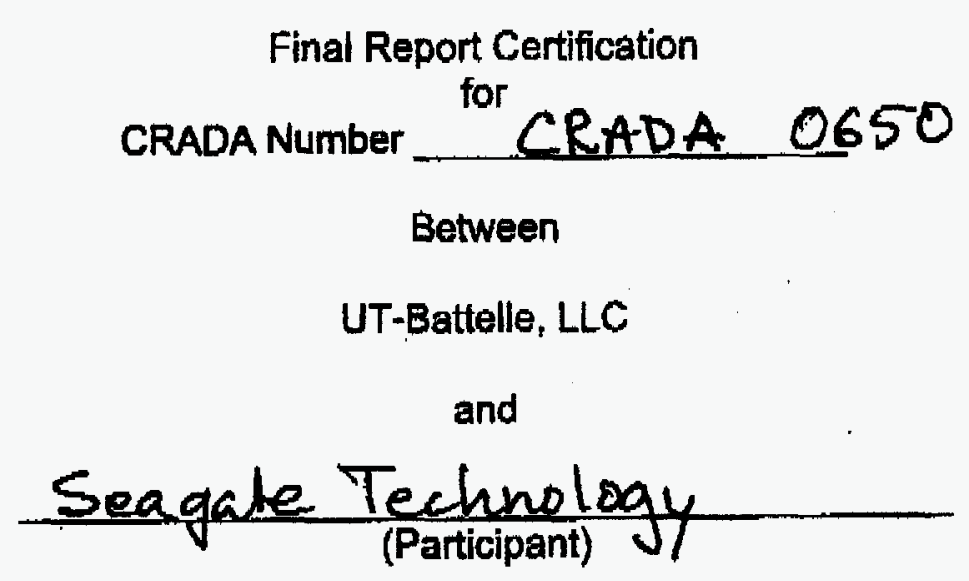

Instructions:

Mark the appropriate statement in 19 or 16 below with an 'IX." Refer to the articles in the CRADA terms and conditions governing the identification and marking of Protected CRADA Information (PCI).

If no $\mathrm{PCl}$ is identified, the report will be distributed without restriction. If $\mathrm{PCl}$ is identified, the report distribution will be limited in accordance with the CRADA terms and conditions goveming release of data. In all cases items 2 and 3 must be true. That is, the report cannot contain Proprietary information and a disclosure must be filed prior to release of the report.

This certification may either be made by using this form or may be made on company letterhead if the Participant desires. A faxed copy of this completed form is acceptable.

The following certification is made for the subject final report:

1. (a) The final report contains information that qualifies as "Protected CRADA information" (PCl). The PCl legend is printed on the report cover, and the $\mathrm{PCl}$ is clearly identified.

OR

IX (b) The final report does not contain "Protected CRADA Information." The "Approved for Public Release" legend is printed on the report cover.

2. The final report does not contain Proprietary Information.

3. By the signature below, the Participant has no objection to the public distribution of the final report due to patentable information.

For the Participant:

$\frac{80 u 20}{\text { (Name) }}$
$\frac{6 / 27 / 07}{\text { (Tike - Pesident R s }}$
(Date)




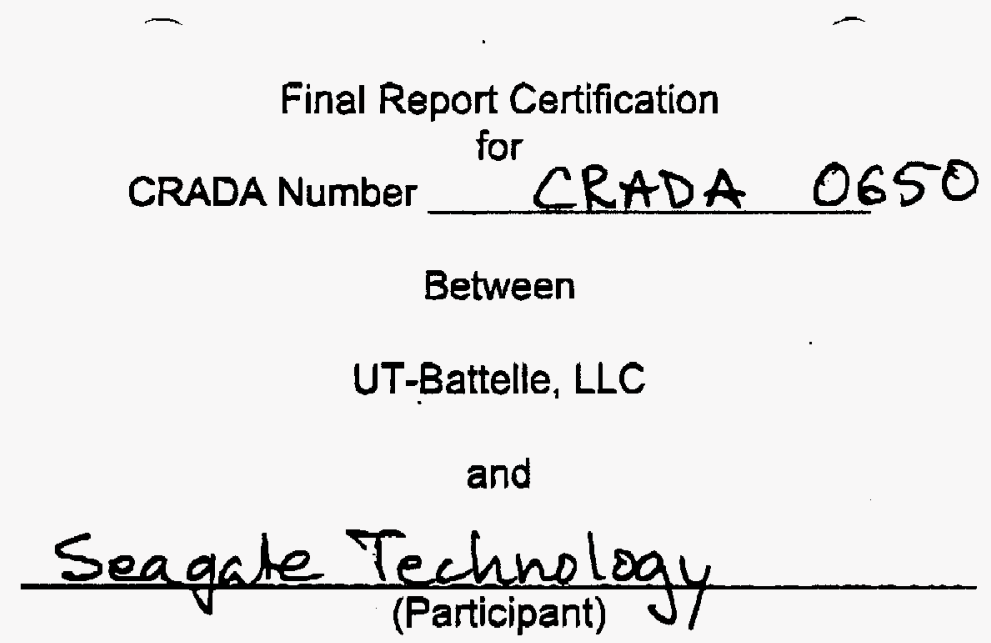

\section{Instructions:}

Mark the appropriate statement in 1a or $1 \mathrm{~b}$ below with an 'IX." Refer to the articles in the CRADA terms and conditions governing the identification and marking of Protected CRADA Information (PCl).

If no $\mathrm{PCl}$ is identified, the report will be distributed without restriction. If $\mathrm{PCl}$ is identified, the report distribution will be limited in accordance with the CRADA terms and conditions governing release of data. In all cases items 2 and 3 must be true. That is, the report cannot contain Proprietary Information and a disclosure must be filed prior to release of the report.

This certification may either be made by using this form or may be made on company letterhead if the Participant desires. A faxed copy of this completed form is acceptable.

The following certification is made for the subject final report:

1. (a) The final report contains information that qualifies as "Protected CRADA Information" (PCl). The PCl legend is printed on the report cover, and the $\mathrm{PCl}$ is clearly identified.

OR

IX (b) The final report does not contain "Protected CRADA Information." The "Approved for Public Release" legend is printed on the report cover.

2. The final report does not contain Proprietary Information.

3. By the signature below, the Participant has no objection to the public distribution of the final report due to patentable information.

For the Participant:

$\frac{\text { Leude }}{\text { (Name) }}$
$\frac{\text { Vice - President } R \xi \Delta}{\text { (Title) }}$
$\frac{6 / 27 / 07}{\text { (Date) }}$




\section{Seagate Technology}

1 Disc Drive

Bloomington, MN 55435

Telephone: $\quad 952-402-7865$

DATE: $\frac{6 / 28 / 01}{\text { Robert Sco.t }}$

FROM:

Olle Heinanen

NUMBER OF PAGES (Including this page):

Urgent

Confidential

The information contained in this facsimile message may be privileged and confidential. It is intended only for the use of the individual or entity to whom it is sent: If the recipient of this transmittal is not the intended recipient, or an employee or agent responsible to deliver it to the intended recipient, any dissemination, distribution or copying of this communication is strictly prohibited. If you have received this communication in error, please immediately notify us by telephone and return the original message to us at the above address via the Postal Service. Seagate will reimburse you reasonable telephone and postal expenses for doing so. Thank you. 
FINAL REPORT

CRADA Project Number: ORNL 01-04

\title{
Fundamental Scientific Problems in Magnetic Recording
}

\author{
Thomas C. Schulthess \\ Phone: 865-574-4344 \\ Fax: 865-241-0381 \\ schulthesstc@ornl.gov \\ Computer Science and Mathematics Division, ORNL \\ Michael K. Miller \\ Phone: $865-574-4719$ \\ Fax: $865-241-3650$ \\ millermk@ornl.gov \\ Materials Science and Technology Division, ORNL.
}

Project Start: Fall 2001 (interrupted in 2002)

DOE Project Funding:

FY-01:

FY-02:

$\$ 40 \mathrm{~K}$

FY-03:

$\$ 0 \mathrm{~K}$

FY-05: $\quad \$ 210 \mathrm{~K}$ 


\section{Abstract}

Magnetic data storage technology is presently leading the high tech industry in advancing device integration - doubling the storage density every 12 months. To continue these advancements and to achieve terra bit per inch squared recording densities, new approaches to store and access data will be needed in about three to five years. In this project, collaboration between Oak Ridge National Laboratory (ORNL), Center for Materials for Information Technology (MINT) at University of Alabama (UA), Imago Scientific Instruments, and Seagate Technologies, was undertaken to address the fundamental scientific problems confronted by the industry in meeting the upcoming challenges. The areas that were the focus of this study were to: (1) develop atom probe tomography for atomic scale imaging of magnetic heterostructures used in magnetic data storage technology; (2) develop a first principles based tools for the study of exchange bias aimed at finding new anti-ferromagnetic materials to reduce the thickness of the pinning layer in the read head; (3) develop high moment magnetic materials and tools to study magnetic switching in nanostructures aimed at developing improved writers of high anisotropy magnetic storage media. 


\section{Principal Project Personnel}

Role in project:

Areas of research:

Role in project:

Areas of research:

Role in project:

Areas of research:

Role in project:

Areas of research:

Role in project:

Areas of research:

Role in project:

Areas of research:
Thomas C. Schulthess (ORNL)

Lead theory part of project, development of ab-initio based theory of exchange bias and conceptual development of ab-initio Monte Carlo.

Electronic structure theory and multi-scale modeling of magnetic heterostructures; theory of exchange bias.

Michael K. Miller (ORNL)

Lead development of atom probe tomography.

Atom probe tomography characterization of multilayer thin films.

\section{G. Malcolm Stocks (ORNL)}

Develop ab-initio Monte Carlo in objective 3.

Methods for large scale electronic structure calculations of general noncollinear magnetic states (co-inventor of constrained local moment method).

William (Bill) H. Butler (MINT, UA)

Theory and calculations for high moment magnets.

Electronic structure theory and magnetism of magnetic recording.

Thomas F. Kelly (Imago Scientific Instruments)

Development of local electrode atom probe and specimen preparation methods for multilayer thin films.

Local electrode atom probe characterization of thin films.

Mark Kief (Seagate Technologies)

Deposition of multilayer thin films.

Recording devices. 


\section{Project Overview}

\subsection{Specific objectives}

Few industries have advanced as rapidly over the past two decades as the magnetic recording industry. The density at which information is stored on a magnetic disk drive has been growing super-exponentially during the past decade. Compound annual growth rates for bit densities were 60 percent in the early $1990 \mathrm{~s}$. Recently, they have exceeded 100 percent. This uniquely torrid pace of technological advance is driven by a fierce international competition. It has become clear to the industry that the projected growth in recording densities confronts fundamental physical problems that cannot be solved by trial and error alone, but call for systematic understanding of the underlying physics.

As recording densities keep increasing, the relevant length scales of features in the underlying devices rapidly approach the nanometer scale. The magnetic bits (approximately $70 \mathrm{~nm} \times 900 \mathrm{~nm}$ for a $10 \mathrm{Gbit} / \mathrm{in}^{2}$ medium, or $30 \mathrm{~nm} \times 200 \mathrm{~nm}$ for $100 \mathrm{Gbit} / \mathrm{in}^{2}$ storage) consist of assemblies of nanoparticles. The reader (Fig. 1) must be small enough (approximately the size of the bit) in order to be sensitive to one bit at a time. At the same time, it must be extremely sensitive to magnetic fields in order to detect the magnetization of the bit. Modern read sensors utilize magnetic multilayers to sense these small magnetic fields.

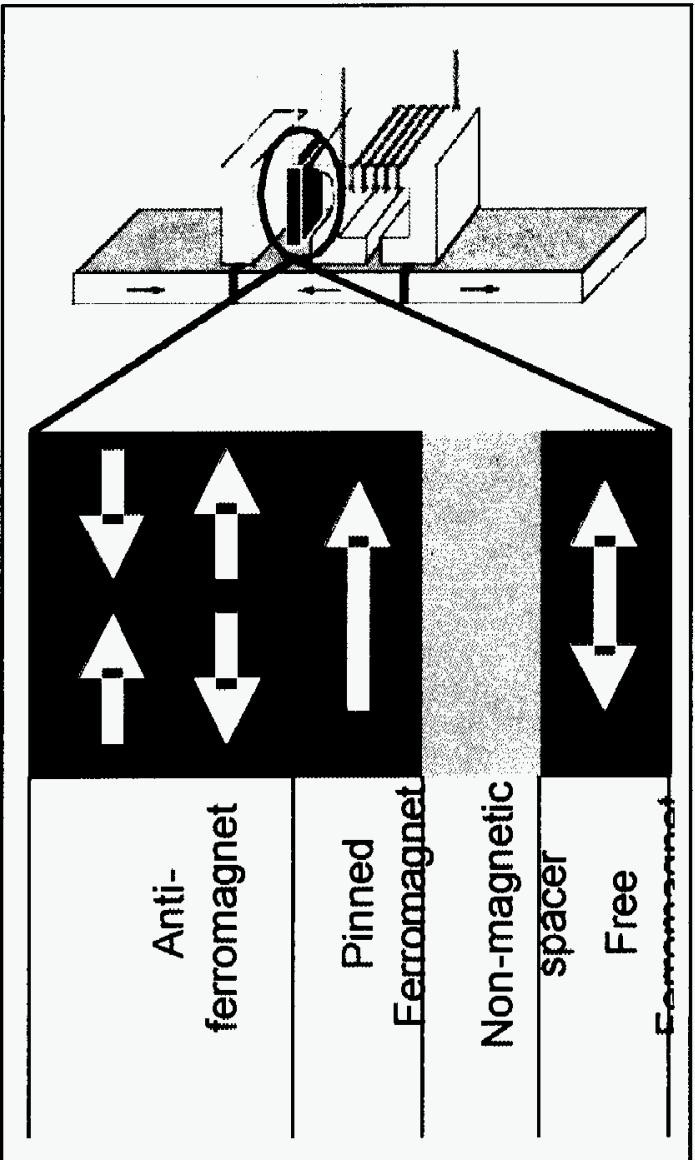

Fig. 1: Cartoon of magnetic recording head for longitudinal recording. They typically consist of two ferromagnetic layers separated by a spacer layer. The in-plane resistance of the multilayers depends on the relative orientation of the magnetic moments in the two magnetic layers. In operation, the moments of one of the magnetic layers (called the pinned layer) is held fixed by the adjacent antiferromagnet while the moments in the other layer (called the free layer) are free to rotate in response to an applied magnetic field. The total thickness of the magnetic layers and the spacer layer may be only $10 \mathrm{~nm}$. The free layer typically is a composite layer consisting of Co and permalloy $\left(\mathrm{Ni}_{80} \mathrm{Fe}_{20}\right)$, which makes the layer magnetically soft. The cobalt layer, which is deposited at the interface of the free layer to enhance the magnetoresitive effect, may be only one or two atomic layers in thickness. A major objective is to obtain the largest possible change in resistance for relatively small changes in the magnetic field. One key to achieving this objective is to grow films with the proper interfacial structure. It is important to avoid roughness on a relatively long scale $(10 \mathrm{~nm})$ in order to optimize the magnetic performance of the device'. 
Moreover, it is important to avoid roughness on the atomic scale in order to maximize the electrical response of the reader. ${ }^{\text {i }}$

Recording density of $100 \mathrm{Gbit} / \mathrm{in}^{2}$ has been demonstrated by Seagate earlier this year, but present technology will only allow for one or at most two further doublings of the density. To achieve Tbit/in ${ }^{2}$ storage, new materials have to be introduced and different approaches have to be used. For example, as the bit size decreases, the limiting factor in the read head is the thickness of the antiferromagnetic layer. Further decrease of the thickness will require antiferromagnets with higher anisotropies, but where pinning field and blocking temperature of the ferroantiferromagnet component of the spin-valve remain unchanged. The even bigger challenge is related to the writing process. As the bit size decreases, materials with higher anisotropies have to be used for the storage medium. This is needed to keep the energy barrier large enough to prevent the magnetization in a bit from reversing, in order that the stored information remains stable at ambient temperature over the lifetime of the storage medium. Materials for $\mathrm{Tbit} / \mathrm{in}^{2}$ storage, such as assemblies of FePt nanoparticles, are presently known. However, it is presently unclear how information can be written (the magnetization of the bit reversed deliberately) into storage media made from these materials. Writing such high anisotropy media will require new high moment materials in the writer and more sophisticate writing process. Seagate, for example, is presently pursuing the option of thermally assisting the writing process.

The objective of this project was to address the fundamental science that underlies these technological challenges and to clear the path forward for the development of $\mathrm{Tbit} / \mathrm{in}^{2}$ storage technology. As the properties of magnetic nanostructure are dominated by interface magnetism, the properties cannot be inferred from bulk parameters, but have to be calculated for the actual system from the first principles based calculations. Such calculations, however, can only lead to meaningful results if chemical composition and atomic structure of the heterostructures are known. The project consisted of four specific tasks:

1. Develop atom probe tomography as a technique to determine the atomic structure of magnetic multilayers and nanoparticles.

2. Develop first principles based models for exchange bias and perform calculations for systems that are prototypical for magnetic recording applications.

3. Develop first principles based models to study the switching of magnetic nanoparticles.

Task 3 was not originally part of this project and was added because understanding the switching in magnetic nanoparticles has become a high priority problem in the industry.

\subsection{Relationship to DOE mission and other DOE funded projects}

All the research performed in this project is closely related to other DOE funded projects. The development of atom probe tomography in Materials Science and Technology Division (formally Metals and Ceramics Division) at ORNL is partially funded by BES/DMSE. Similarly, synthesis, characterization, and modeling of FePt nano-particles are the primary subject of a new BES/DMSE funded Field Work Proposal. In this project, we leveraged this new BES project and focused on calculating the properties of these nano-particles that are of direct relevance to magnetic data storage. Furthermore, the first principles electronic structure codes and methods, which all models used in this project are based, are being developed with ASCR/MICS and BES/DMSE funding, making efficient use of new high performance computing infrastructure at ORNL's Center for Computational Science. Putting the new high performance computers to work solving real problems relevant to science and technology that immediately impact society and our

\footnotetext{
${ }^{1}$ It should be noted that the dependence of giant-magnetoresistance on the interfacial structure is still not fully understood. There is evidence that in some systems, interfacial roughness may even enhance the effect. See, for example, R. Schad, P. Beliën, G. Verbanck, V. V. Moshchalkov, and Y. Bruynseraede, H. E. Fischer, S. Lefebvre and M. Bessiere, "Giant Magnetoresistance dependence on the lateral correlation length of the interface roughness in magnetic superlattices," Physical Review B 59, 1242 (1999).
} 
economy are primary objectives for the new ultra scale simulation initiative. This initiative, in turn, is funding the high performance-computing infrastructure needed to perform the calculations in the present project.

\section{Scientific and Technical Approach}

The major research activities are in three areas: (1) the development of atom-probe tomography; (2) the development and use of first principles based models for ferro-antiferromagnet bilayers, and high moment materials; and (3) the development of ab-initio Monte Carlo methods to calculate temperature dependent free energy barriers for magnetization reversal of grains and nanoparticles.

\subsection{Atom-probe tomography}

Because of the extremely short range of the exchange interaction and of the scattering processes involved in electron transport, it is necessary to know the structure of magnetic multilayer films at the atomic length scale if one is to understand and rationally optimize their properties. Thus, one of the greatest impediments to understanding and optimizing magnetic multilayer films has been our inability to determine the structure of these polycrystalline, nanometer scale films with the necessary atomic resolution. Recently, however, atomic scale resolution has been achieved by atom probe tomography ${ }^{\text {iii. }}$. In this technique, specimens are fabricated into needles with end radii of curvature less than $50 \mathrm{~nm}$. A pulsed electrical potential is applied to the specimen to produce a sufficiently high electrical field at the tip to ionize and remove atoms. The atoms are collected in a time-of-flight mass spectrometer equipped with a position-sensitive detector that encodes their $\mathrm{x}$ - and $\mathrm{y}$-coordinates. The mass-to-charge state ratio and hence the elemental identity of each ion is determined from its flight time from the specimen to detector. The resulting data enables the reconstruction of a three-dimensional image of the specimen with atomic scale resolution. This technology is well developed for bulk conducting specimens ${ }^{\text {iv }}$, but is still in its infancy for magnetic multilayer films. In this project, we have developed techniques that will extend atom probe tomography to magnetic multilayer films of interest to the recording head industry and then to use the information obtained about interface structure to optimize the read sensors.

It will be clear that success in this part of our project will have a major, positive impact on other parts of the project as well as on many other aspects of magnetic recording. The ability to obtain atomic scale images of nanostructured materials will be very important for understanding the exchange bias problem which depends so sensitively on the physical and chemical structure of the interface. Similarly, as we attempt to engineer atomic scale structures with higher saturation magnetization, it will be crucial to know if we have, in fact, made the structure that theory predicts will have the desired properties.

\subsection{First principles based models of ferro-antiferromagnet bilayers}

Exchange bias refers to a shift in the magnetization curve (MH-loop) of a ferromagnetic material (FM) that is in contact with an antiferromagnet (AFM) ${ }^{v}$. This shift is used as a bias to pin one of the magnetic layers in the read sensor. Three issues are crucial for the performance of the sensors: First, the magnitude of the shift should be as large as possible: Second, the blocking temperature above which the shift vanishes, should be as high as possible: Third, the amount of AFM material required to achieve a stable bias should be as small as possible.

Despite four decades of research since its discovery, there are still significant gaps in our understanding the microscopic origin of exchange bias. This has made the search for materials to optimize the above-mentioned properties a matter of trial and error and quite inefficient in view 
of the large number of parameters that are involved (e.g., different growth techniques, different AFM materials, and different interface preparations).

Significant progress has been made recently in understanding effects that are observed along with exchange bias, such as the enhanced coercivity ${ }^{\mathrm{vi}}$. Model exchange bias systems have even been produced in which the coupling between the FM and the AFM material can be modeled straightforwardly ${ }^{\mathrm{vii}}$. However, for the class of AFM materials that is relevant to applications in magnetic recording, the origin of exchange bias is still a puzzle. This is because most of these systems have AFM/FM interfaces that are compensated on the AFM side; i.e., the net moment of the AFM side of the interface is zero when averaged over the entire sample size. The most straightforward interpretation of such a situation would be the exchange between the FM and the AFM averages to zero so that there would be no exchange bias. To resolve this problem, Malozemoff ${ }^{\text {iii }}$ proposed a model, the random field model, in which the AFM breaks up into domains, as a result of which, the net AFM/FM exchange averaged over the domain size is different from zero. The validity of this proposition has been checked experimentally ${ }^{\text {ix }}$ and the model is accepted as one way to explain exchange bias.

Another coupling mechanism between AFM with compensated interface planes and the FM was given by Hinchey and Mills ${ }^{\mathrm{x}}$, as well as independently by Koon ${ }^{\mathrm{x}}$. They showed that the frustration at the interface forces the FM magnetization to align perpendicular to the AFM magnetization axis. This mechanism is called spin-flop coupling, since it is similar to the wellknown spin-flop state of an AFM in a strong magnetic field. The perpendicular alignment of the FM to the AFM moments has been confirmed experimentally ${ }^{x i i}$ for several AFMs. However, the spin-flop coupling that leads to perpendicular coupling are uniaxial rather than unidirectional and therefore can not account for exchange bias ${ }^{\text {xiii }}$. Furthermore, since this coupling is perpendicular to any net uncompensated coupling within Malozemoff's model, the spin-flop mechanism will actually reduce the exchange bias. As Stiles and $\mathrm{McMichael}^{\mathrm{xiv}}$ pointed out; for any reasonable AFM grain or domain size, the spin-flop coupling mechanism will be stronger than the unidirectional coupling mechanism in Malozemoff's model and it is then no longer clear why there should be any exchange bias. We are, therefore, left with a paradox: exchange bias is observed. Malozemoff's model (which is experimentally verified) can explain it, but the spinflop coupling mechanism (which is also verified experimentally) does not yield exchange bias and renders the unidirectional coupling in Malozemoff's model irrelevant.

There are two primary reasons for this paradox as well as the persistent difficulty of building a predictive theory of exchange bias: (1) apart from a few model systems, little to nothing is known about the interfacial structure and exchange of FM/AFM bilayers; and (2) predictive models of exchange bias will have to include atomic length scale (interfacial exchange) but have to cover physics at the length scales larger than the AFM domains and grains (100s of $\mathrm{nm}$ of $\mu \mathrm{m}$ ).

Despite four decades of research, a predictive theory of exchange bias is missing. It would be unreasonable to expect from the present project a final and definitive word on exchange bias. However, given the remarkable progress that has been made in recent years towards the understanding of the physics of AFM/FM bilayers at longer length scales, we anticipate that with the present project, we will fill in the missing links for a predictive theory of exchange bias. We have three arguments in our favor: (1) the development of atom probe tomography (see previous section and progress report below) will allow us to access structural information of the layered heterostructures at the atomic scale; (2) our capabilities to perform all electron ab-initio electronic structure calculations for systems with thousands of atoms and in the context of effective medium theories for thousands of atomic layers will allow us to make definite predictions about the magnetic exchange at the interface; (3) our ability to map electronic structure results onto simple spin models (see progress report below) and to perform atomistic simulations of spin models over long time scales will allow us to extract the parameters form atomistic simulations needed by 
models that cover the long length scale physics (for example, uniaxial exchange between FM and AMF grains even when spin-flop coupling dominates).

The research plan of this part of the project is to perform ab-initio electronic structure calculations for typical AFM and AFM/FM bilayers with existing codes, develop ways to map the electronic structure onto Heisenberg spin-models in the context of effective medium theories needed to describe random alloys (most AFM and FM used in magnetic recording are random alloys), and investigate the specific nature of exchange between AFM/FM interfaces. In phase II, we expand our electronic structure methods to cover the relevant physics we determine necessary to describe the exchange bias effect - this includes both the actual ab-initio calculations as well as the mapping onto simpler spin models. In phase III, we use the atomistic structure determined experimentally with atom-probe tomography for the AFM/FM systems used in magnetic recording to calculate the effective exchange between FM and AFM grains using a combination of ab-initio methods, mapping of electronic structure onto simpler spin modes, and fast spin dynamics simulations.

\subsection{Ab-initio Monte Carlo methods}

Present ab-initio methods are capable of predicting ground state properties of magnetic materials, i.e., they allow us to calculate the zero temperature internal energy barrier, $\Delta E(T=0 K)$, for magnetization reversal. However, the quantity that is needed to study the switching of nanoparticles or to establish effective coupling in small ferro-antiferromagnet grains is the temperature depended free energy barrier, $\Delta F(T)=\Delta E(T)-T \Delta S(T)$. For most materials and temperature ranges of interest, the magnetic moment is well formed and the Fermi function is close to a step function. We can, therefore, neglect the effect of electron hole pair excitations and consider only orientational contribution to the entropy. Furthermore, the internal energy can be calculated with existing zero temperature electronic structure methods, $\Delta E(T) \approx \Delta E(T=0)$. It is thus only necessary to sample various orientational configurations, $\left\{\vec{e}_{i}\right\}$, of the atomic moments of nanoparticles or grains. In this project, we will use the well-developed Monte Carlo methods to simulate classical spin systems, but where we calculate the energy, $E\left[\left\{\vec{e}_{i}\right\}\right]$, of every moment configurations from first principles using the constrained local moment method (CLM). We call this Ab-initio Monte Carlo, which we will implement AIMC in two ways; 1) using regular Metropolis based sampling; and 2) using the novel "Wang-Landau" sampling algorithm.

Metropolis type sampling: In order to equilibrate a magnetic state in the nanostructures at a certain temperature, we will sample the space of magnetic configurations using Metropolis based methods, where a trail moves from an initial configurations $\left\{\vec{e}_{i}\right\}$ to $\left\{\vec{e}_{i}\right\}$, and is accepted with probability $p=\min \left(1, \exp \left(-\left(E\left[\vec{e}_{i}^{\prime}\right]-E\left[\vec{e}_{i}\right]\right) / k_{B} T\right)\right)$. Conceptually, this is rather simple. However, because $E\left[\left\{\vec{e}_{i}\right\}\right]$ is computationally every expensive to evaluate, we will have to study various techniques (e.g., cluster updates method, over relaxation method and others[77]) to minimize the number of trial moves needed to reach thermal equilibrium. All these techniques have been developed for simple models and it will be a research task to find out what their properties are when the underlying energy expression is replaced by a full electronic structure calculation.

Wang Landau Sampling: In order to calculate the free energy with Metropolis based Monte Carlo methods, one would have to repeat the first principle calculations at every temperature. Since we limit ourselves to sampling only orientational contributions to the entropy, 
the free energy takes the form $F=E-k_{B} T \log (W(E))$ where $W(E)$ is the density of states (DOS) in the space of magnetic configurations. Note that $W(E)$ is temperature independent. We propose to use the novel "Random walk in energy space with a flat histogram" method, also known as "Wang-Landau Sampling," to calculate $W(E)$. With this approach, all electronic structure calculations can be performed at zero temperature, but the free energy and other thermodynamic quantities can be obtained at all temperatures without much additional computational effort. To calculate the DOS, one starts with an initial guess for $W(E)=1$ and accepts trial moves with probability $p\left(E\left[\left\{\vec{e}_{i}\right\}\right] \rightarrow E\left[\left\{\vec{e}_{i}\right\}\right]\right)=\min \left(W\left(E\left[\left\{\vec{e}_{i}\right\}\right]\right) / W\left(E\left[\left\{\vec{e}_{i}\right\}\right]\right), 1\right)$. Every time a move is accepted, the DOS is updated with $W(E) \rightarrow W(E) * f$ where $f>1$. These updates are continued until all energies have been visited equally often (until the histogram is flat). The factor $f$ is then reduced and the procedure restarted with the DOS from the previous iteration as an initial guess. This algorithm results in a self-avoiding random walk in energy space and the simulations thus do not suffer from the usual problem of being trapped in local minima.

\section{Results}

\subsection{Atom-probe tomography}

A primary initial objective of this project was to develop Atom Probe Tomography (APT) as a reliable and consistent technique for atomic-scale imaging of magnetic multilayers and their interfaces. An outline of one method that was developed to fabricate needle-shaped atom probe specimens from flat multilayer films is shown in Fig. 2. The multilayers were deposited onto square (or round) silicon posts prepared with the Bosch process. Individual posts containing the multilayer were cut from the wafer and carefully mounted onto a blunt tungsten or stainless steel needle. Alternatively, a method in which a coupon consisting of a $6 \times 6$ array of posts that could attach to a special specimen holder was also developed. In both methods, these posts were milled in a dual beam focused ion beam (FIB) instrument with the use of an annular pattern of $30 \mathrm{keV}$ gallium ions. The inner and outer diameters of the beam were adjusted during milling to produce the desired end form in the minimum amount of instrument time. This method produced needleshaped specimen with an end radius of less than $50 \mathrm{~nm}$.

One of the problems encountered during initial atom probe tomography experiments on multilayer films was the implantation of gallium into the specimen, as shown in Fig. 3. Gallium levels of up to 30 percent were experimentally observed in the atom probe analyses, particularly at the exposed surface of the specimen. This implantation was especially a problem when the gallium ion beam was used to image the specimen. These high levels of gallium implantation were shown to intermix the layers and introduce a large strain into the lattice that caused premature specimen failure. Therefore, the FIB-based specimen preparation method was refined to minimize gallium implantation. An additional step of depositing an $\sim 2$ - m-thick protective platinum cap onto the surface of the multilayer in the FIB, Figure 2, prior to annular ion milling, was found to reduce the gallium levels in the specimens to less than 0.5 percent. The resulting method provides a similar proportion of successful analyses as traditional metallic specimens.

The influence of the resistivity of substrate was investigated with high resistivity ( $5 \Omega$ $\mathrm{cm})$ and low resistivity $(0.05 \Omega-\mathrm{cm})$ silicon substrates. The mass spectra from multilayers deposited on the high resistivity substrates differ from those from metallic specimens in that the sharp mass peaks have a pronounced high mass tail which degrade the mass resolution and complicate the estimation of the concentrations due to peak overlap. These high mass tails are due 
to large energy deficits of the field-evaporated ions. These large energy deficits are indicative of a significant reduction of amplitude of the high voltage pulse used to initiate field evaporation due to poor electrical conduction. The background noise level was significantly higher than in metallic specimens. The background noise also decreased with the elapsed time during the experiment and was higher in the Ti layers compared to the $\mathrm{Nb}$ layers, Fig. 4. The background noise is due to a combination of the dark count of microchannel plates in the detector plus some other sources of noise and to unsynchronized field evaporation of the specimen on the standing voltage. The decrease in the number of lost ions with time is manifested in an apparent change in thickness of the films with distance, especially during the initial stages of the experiment, Fig. 4. The uncontrolled field evaporation was more prevalent in the $\mathrm{Ti}$ layers than in the $\mathrm{Nb}$ layers due to its lower evaporation field. The change in the amount of uncontrolled field evaporation with time is most likely due to a change in the temperature at the analysis surface of the multilayers due to poor thermal and electrical conduction. These effects were not observed on low resistivity $(0.05 \Omega-\mathrm{cm})$ silicon substrates.
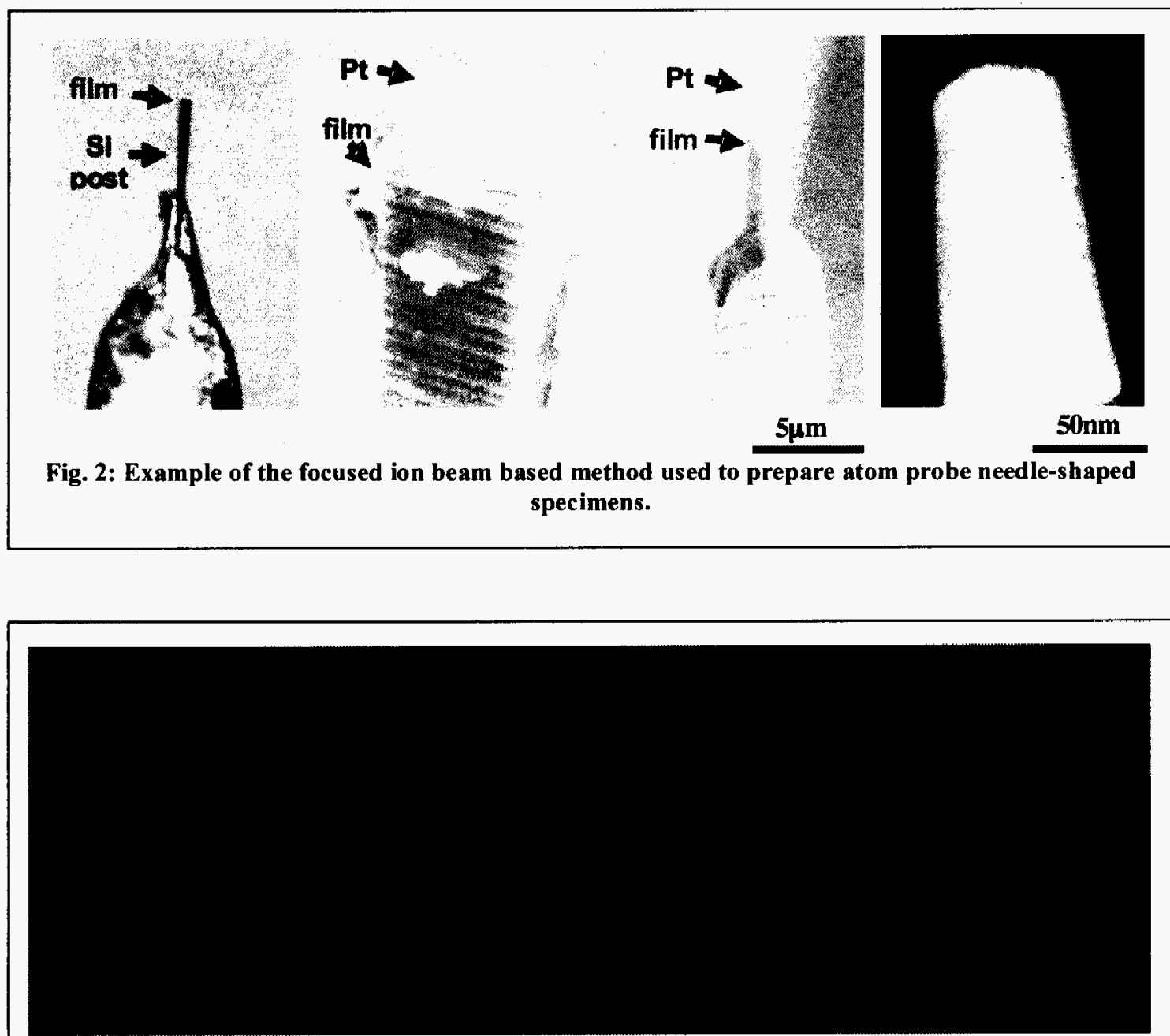

Fig. 3: Example of undesired gallium implantation into the specimen as a result of the focused ion beam milling method used to fabricate the atom probe specimen.

Some selected examples of atom maps and their associated concentration profiles that were collected from $\mathrm{Cu}-\mathrm{Co}$ and four bilayer repeats of a PtMn $(150 \mathrm{~nm})-\mathrm{CoFe}(25 \mathrm{~nm})$ multilayer 
are shown in Figs. 5-8. In suitably oriented specimens, the individual lattice planes can be detected as shown in the $\mathrm{Cu}$-Co multilayer in Fig. 5. The power of atom probe tomography to reveal the distribution of solute at the atomic scale is illustrated in detecting carbon segregation to the interface between the layers in the PtMn-CoFe multilayers, as shown in the atom maps in Fig. 7 and the corresponding concentration profiles constructed normal to the interface in Fig. 8.

These results have indicated that the local electrode atom probe is a highly useful tool in the analysis of such structures. Continued improvement in the technology used in atom probe tomography and in specimen preparation will continue to yield significant advances. In particular, the use of laser pulsing to assist in the field evaporation of atoms from the specimens will enable the technique to be applied to materials with significantly lower electrical conductivities. This advance will enable the technique to be applied to a wider range of semiconducting materials. The FIB-based specimen preparation techniques developed in this project are already being adapted to other types of material geometries as well as other materials and will enable site specific specimen preparation to be performed. This improved analysis capability may prove to be vital link for full optimization of data storage structures (compared to theoretical predictions) and the achievement of true atomic level engineering of magnetic thin films. 

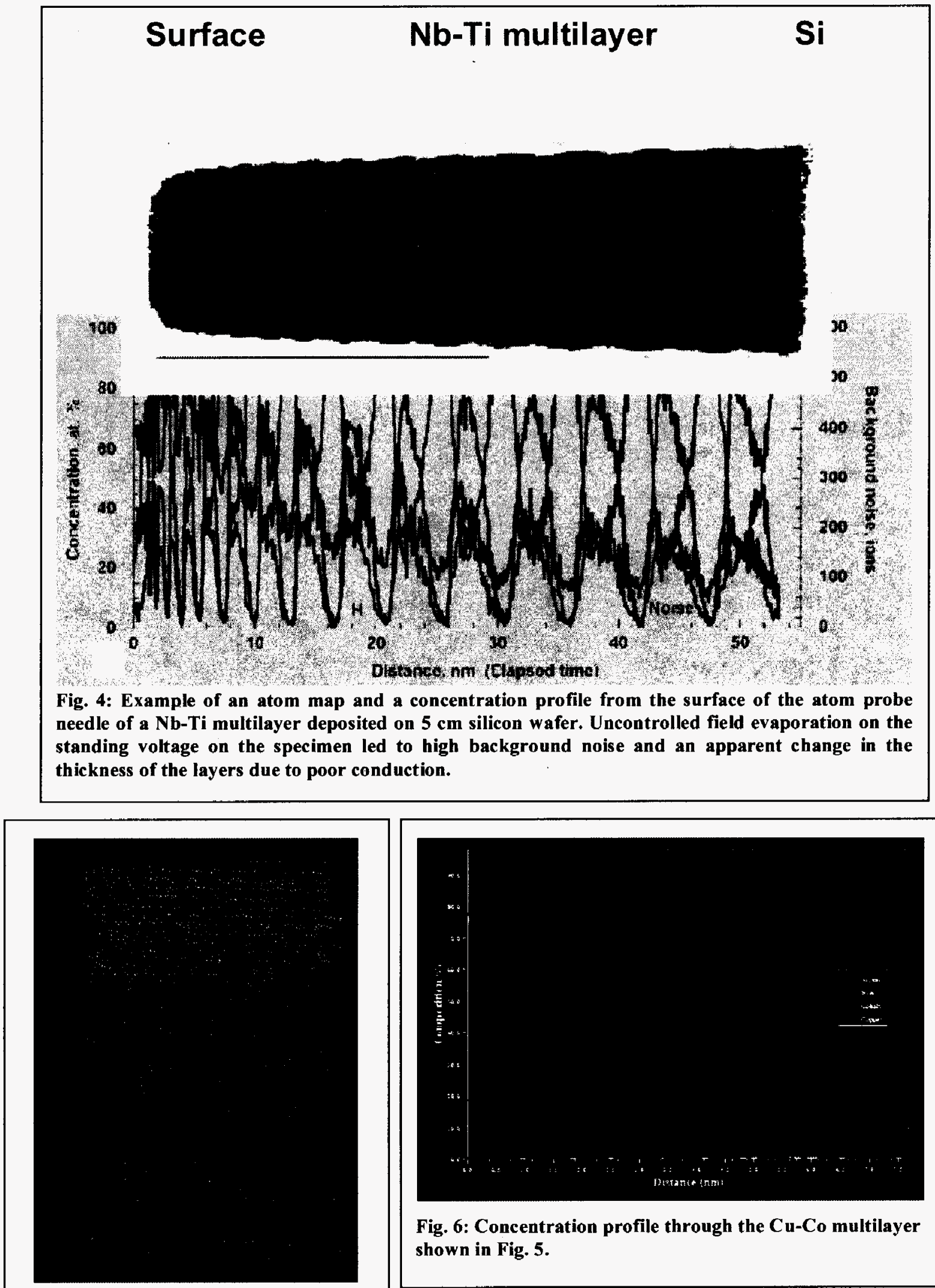

Fig. 6: Concentration profile through the $\mathrm{Cu}-\mathrm{Co}$ multilayer shown in Fig. 5.

Fig. 5: Atom maps of a Cu-Co multilayer showing crystallographic planes. 


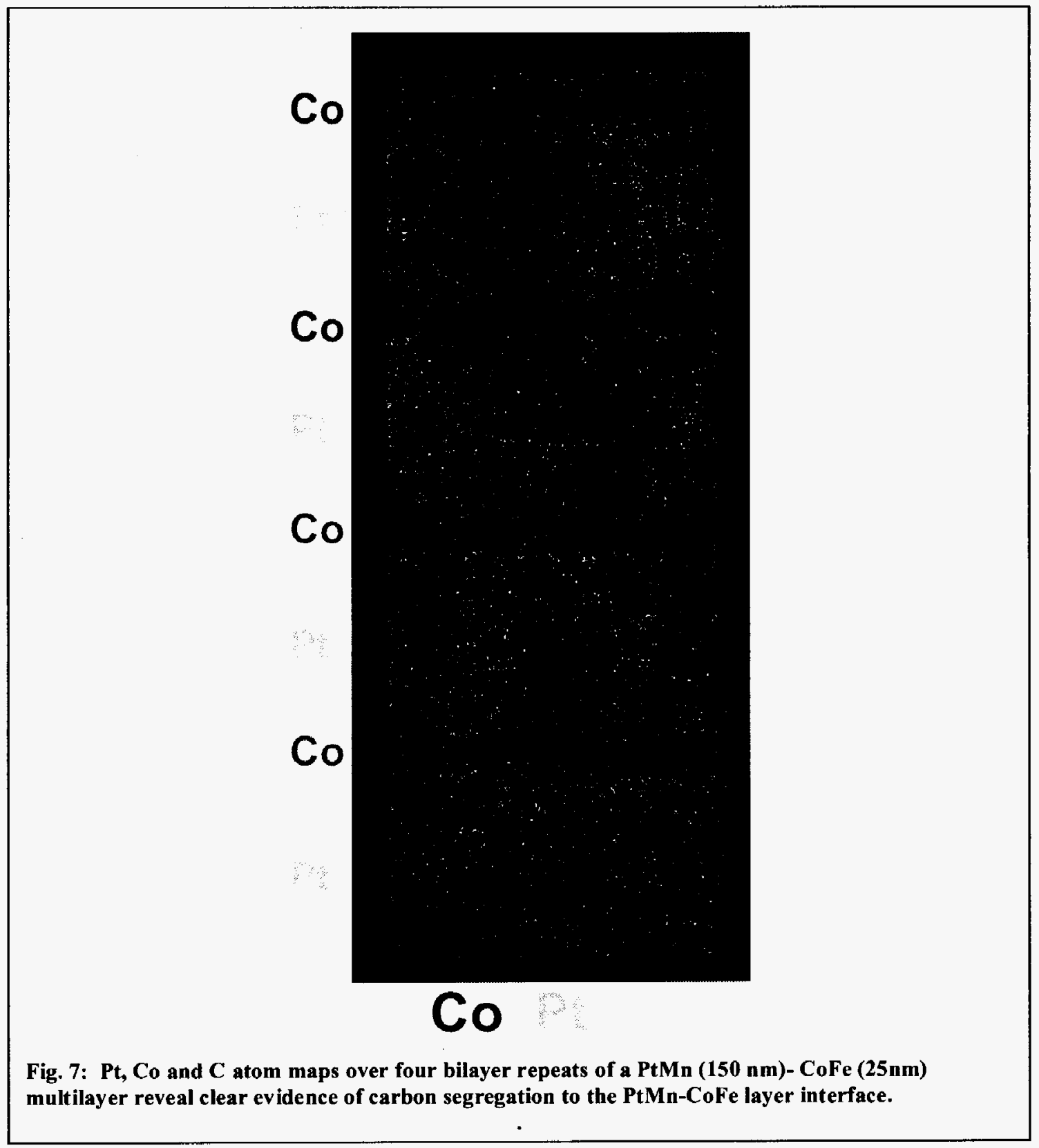

\subsection{Ferro-antiferromagnet bilayers}

The progress we have made to date is along two main lines: (1) building and executing large scale ab-initio simulation for itinerant antiferromagnets as well as ferro-antiferromagnet bilayers; and (2) building ab-initio based spin models and perform large Monte Carlo simulations of ferroantiferromagnet bilayers. 


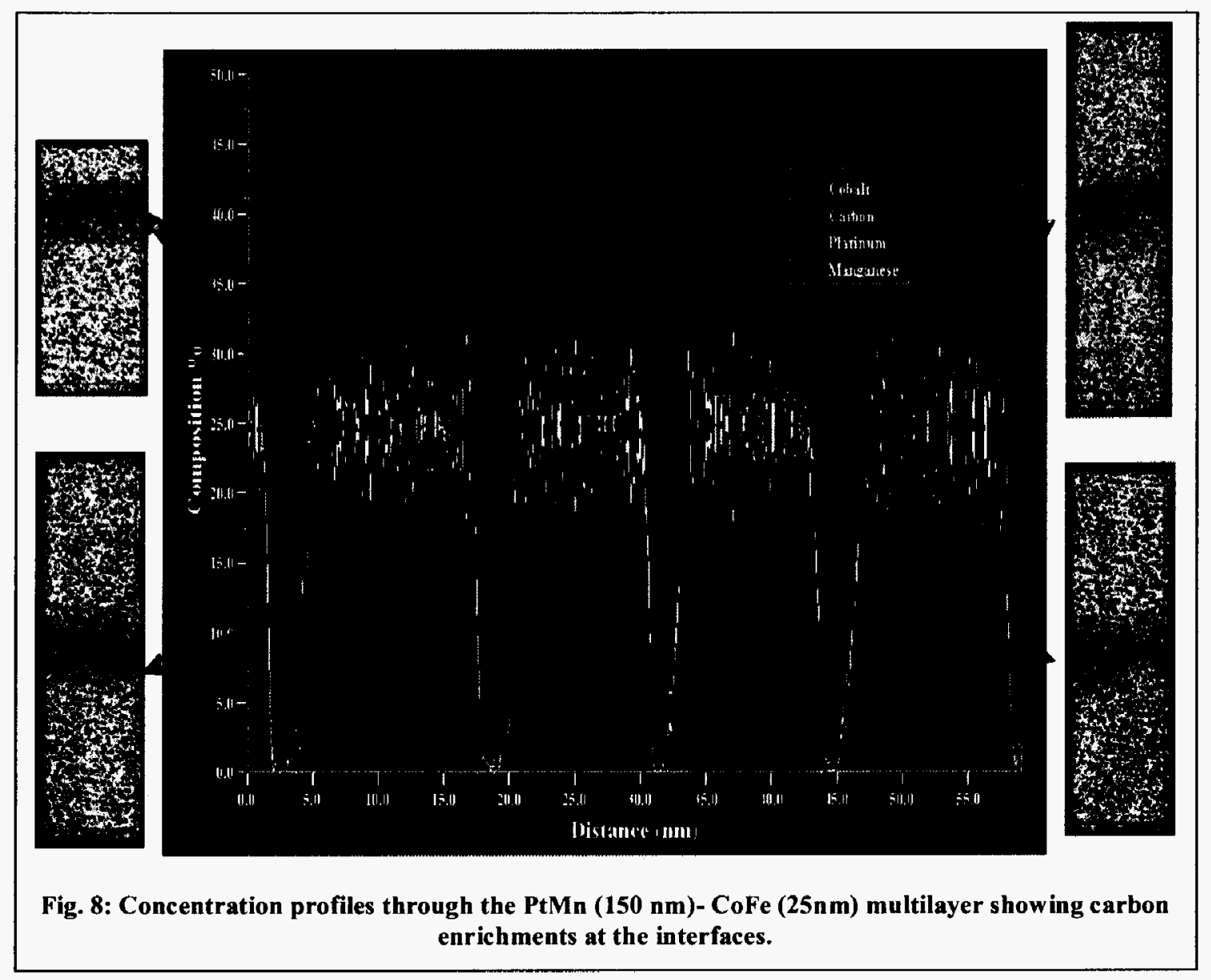

Ab-initio simulations of FeMn and FeMn/Co bilayers: We have used the Constrained Local Moment method to calculate the magnetic ground state of FeMn and FeMn/Co bilayers. In agreement with earlier mean field calculations and with Neutron and Mossbauer experiments, we find that the lowest energy state in FeMn is a $3 Q$ state with the moments of the four fcc sublattices pointing along body diagonals of the cube. We find the moments of Fe and $\mathrm{Mn}$ to be almost the same magnitude and the scattering in orientation to be only a few degrees around the ideal orientation of the $3 \mathrm{Q}$ structure. This explains why neutron scattering experiments see a definite magnetic order, even though the alloy is chemically random and consisting of elements that are magnetically quite different. Having established the non-trivial antiferromagnetic ground state in FeMn, we expanded our calculations to FeMn/Co bilayers. Here, we found a result that was quite surprising. The moments in FeMn, when coupled to Co, assume a 1Q like structure with a principle axis perpendicular to the orientation of the Co moments. Note that in the bulk, the 1Q structure is unstable. The perpendicular alignment is, however, consistent with the spinflop coupling model predicted by simple Heisenberg models.

Spin-models and large scale Monte Carlo simulations: We have performed large scale Monte Carlo simulations of simple spin models of ferro-antiferromagnet bilayers focusing on phase transition of the coupled system. These simulations were motivated by recent experiments by van der Zaag et. al., which showed, that the ordering temperature in the antiferromagnet appears higher than in the bulk, when the film is thin enough and in proximity to the ferromagnet. Note that from finite size scaling arguments one expects (and observes) a lowering of the Neel temperature in free thin films. Our simulations show that the ordering temperature is raised in the antiferromagnet only when the interfacial exchange is uncompensated. In fact, the coupled system 
is found to have only one critical point, which is identical to that of the freestanding ferromagnet layer. For compensated interfaces, we find two critical points with a spin-flop transition at the ordering temperature of the antiferromagnet layer.

We have also made progress with the development of ab-initio based spin models for random alloys. The formalism to extract Heisenberg exchange parameters from ab-initio band structure calculations has been developed by Liechtenstein and co-workers over a decade ago. We have extended this method and developed formulas to calculate Heisenberg exchange for the case of random alloys treated within the coherent potential approximation. The method has been implemented in our multiple scattering codes and is presently being applied to calculate exchange parameters FeCo alloys. We are now in a position to develop ab-initio based model spinHamiltonians for the complex alloy systems used in magnetic recording.

Finally, we have also worked on spin models with anisotropic exchange interactions. This work is based on the realization that inversion symmetry at the interface in a bilayer is broken and that the Dzyaloshinsky-Moria exchange term between moments at the interface is always non-vanishing. With the inclusion of this term the symmetry broken (and exchange bias can be explained) in the case of perpendicular coupling between the ferromagnet and the antiferromagnet. We have also shown that the order of magnitude of the symmetry breaking in a random field Heisenberg model with Dzyaloshinsky-Moria interaction is similar to that of the random field Ising model, i.e., they can be expected to yield reasonable values for the exchange bias. These findings resolve paradox of the random field model in Heisenberg like systems we discussed above. Recognizing the importance of the Dzyaloshinsky-Moria interactions has implications for all further modeling efforts of the project: meaningful electronic structure calculations as well as the mapping onto a spin model have to include spin orbit coupling effects to properly account for the Dzyaloshinsky-Moria term. This is, indeed, what we plan to in phases II and III of the project.

Publications related to the work discussed in this section:

- First Principles Calculations of the Magnetic Structure in FeMn/Co Bilayers, B. Ujfalussy, T. C. Schulthess, and G. M. Stocks, in Computer Simulation Studies in Condensed Matter Physics XV, D. P. Landau, S. P. Lewis, and H.-B. Schuttler Eds., Springer, Berlin 2003, p. 48.

- Effect of interfacial coupling on the magnetic ordering in Ferro-antiferromagnetic bilayers, Shan-Ho Tsai, D. P. Landau, and T. C. Schulthess, J. of Appl. Phys., 93, 8612 (2003).

- Rotating Anisotropies without Superparamagnetic grains in exchange bias systems, T. C. Schulthess, Mat. Res. Soc. Proc. Vol. 746, 31 (2003).

- Phase transitions in ferro-antiferromagnetic bilayers with a stepped interface, D. P. Landau, Shan-Ho Tsai, and T. C. Schulthess, J. of Magn. and Magn. Matter., 272, E817 (2004).

\subsection{Ab inito Monte Carlo methods}

The aim of this task is to develop methods to calculate the temperature dependent free energy barrier for magnetic switching in nanoparticles, such as the FePt or CoPt nanoparticles used in data storage applications. The underlying energy model for this could be a simple Heisenberg model, or a suitable extension, or, ultimately, full ab initio calculations. The entropic contribution to the free energy would be determined in a Monte Carlo simulation and our plan was to use the recently developed Wang-Landau sampling scheme to compute the density of states and from it the entropy. It quickly turned out that the original Wang-Landau algorithm was not entirely suitable for this endeavor and that we would have to generalize it. The original algorithm was designed with the Ising Model in mind, which is a discrete model. In our applications, the models would be continuous. Furthermore, the original algorithm was used to study phase transition and 
hence was setup to compute the density of states as a function of energy only. For our purpose, we would need the joint density of states that is a function of energy and effective magnetic moment of the nanoparticle, as we need the free energy as a function of temperature and magnetization as additional order parameter. Therefore, we had to direct our attention to generalizing the Wang Landau algorithm to compute joint densities of states for continuous models. We accomplished this successfully and the new generalized algorithm was published in Physical Review Letters in the spring of 2006. As we are appending a full reprint of this paper to this report, we will only give a brief summary of the main ideas. The problem with continuous models was solved by using kernel functions with finite support to smear the accepted states in the Monte Carlo procedure over multiple bins of the density of states. The issue with joint density of states was mostly a problem with efficiency. To improve the performance of the algorithm, we added a histogram reweighting step between subsequent Wang-Landau sampling series. As a result the random walkers would be pushed to the boundaries of the density of states and into unexplored regions, which greatly reduced the number of sampling steps needed to converge the density of states.

Publications related to the work discussed in this section:

- Wang-Landau algorithm for continuous models and joint densities of states, C.-G. Zhou, T. C. Schulthess, S. Torbrugge, and D. P. Landau, Phys. Rev. Lett., 96, 120201 (2006).

- Monte Carlo simulations in $\mathrm{NiFe}_{2} \mathrm{O}_{4}$ nanoparticles, G.-G. Zhou, T. C. Schulthess, and D. P. Landau, J. of Appl. Phys. 99, 08H906 (2006).

\section{Summary}

Focused ion beam based specimen preparation techniques were developed to enable the atomic structure of magnetic multilayers and nanoparticles to be determined by atom probe tomography. Several different systems including $\mathrm{Nb}$-Ti and $\mathrm{Cu}-\mathrm{Co}$ multilayers, and bilayers of a PtMn (150 $\mathrm{nm})-\mathrm{CoFe}(25 \mathrm{~nm})$ have been successfully analyzed in the local electrode atom probe. The elemental partitioning between the layers and interfacial segregation was quantified.

$\mathrm{FeMn} / \mathrm{CoO}$ multilayers were studied with ab initio electronic structure based spin dynamics methods. The methods we used reproduced the well know non-collinear $3 Q$ state for the bulk antiferromagnetically ordered state in FeMn. In the FeMn/Co bilayers, the FeMn state transforms to a quasi $1 \mathrm{Q}$ antifferomangnetic state due to the proximity to Co. The coupling between FeMn moments and Co moments is perpendicular - similar to the spinflop state observed in a number of exchange bias system. We have also developed an theory for exchange bias that is based on Dzialishinsky-Moria interactions and show that the resulting ferroantiferromagnetic coupling can be unidirectional even when the ferro and antiferromagnet moments couple perpendicularly.

The Wang-Landau algorithm was generalized and now allows us to compute densities of joint densities of states of multiple variables (temperature, magnetization...) and for systems with continuous degrees of freedom. We demonstrated that our method can be used to compute temperature dependent free energy barriers for magnetic nanoparticles and have applied it to realistic models of FePt. This work has been continued as a user project at the Center for Nanophase Material Sciences by Oleg Mryasov from Seagate Technologies.

\section{References}

1 T. C. Schulthess and W. H. Butler, "Magnetostatic Coupling in Spin Valves: Revisiting Nèel's Formula," Journal of Applied Physics, 87, 5759 (2000). 
I W. H. Butler, X.-G. Zhang, D. M. C. Nicholson, T. C. Schulthess, and J, M. MacLaren, "Giant Magnetoresistance from a Waveguide Effect in Cobalt-Copper Multilayers, Physical Review Letters, 76, 3216 (1996).

1 D. J. Larson, P. H. Clifton, N. Tabat, A. Cerezo, A. K. Petford-Long, R. L. Martens and T. F. Kelly, "Atomic-scale analysis of $\mathrm{CoFe} / \mathrm{Cu}$ and $\mathrm{CoFe} / \mathrm{NiFe}$ interfaces," Applied Physics Letters, 77, $726(2000)$.

1 M. K. Miller, Atom Probe Tomography, Kluwer Academic/ Plenum Publishers, New York (2000).

1 For a review on the subject see: J. Nogues and I. K. Schuller, J. Magn. Magn. Mater. 192, 203 (1999); A. E. Berkowitz and K. Takano, J. Magn. Magn. Mater. 200, 552 (1999).

I M. D. Stiles and R. D. McMichael, Phys. Rev. B 59, 3722 (1999); M. D. Stiles and R. D. McMichael, Phys. Rev. B 63, 4405 (2001); Z. F. Li and S. F. Zhang, Appl. Phys. Lett. 77, 423 (2000); Z. F. Li and S. F. Zhang, Phys. Rev. B 61, R14897 (2000); C. Leigton, J. Nogues, B. J. Jonsson-Akerman, I. K. Schuller, Phys. Rev. Lett 84, 3466 (2000).

1 J. S. Jiang, G. P. Felcher, A. Inomata, R. Goyetter, C. Nelson, and S. D. Bader, Phys. Rev. B $61,9653(2000)$.

I A. P. Malozemofff, J. Appl. Phys, 63, 3874 (1988).

I K. Takano, R. H. Kodama, A. E. Berkowitz, W. Cao, and G. Thomas, Phys. Rev. Lett. 79, 1130 (1997); P. Miltenyi, M. Gierlings, J. Keller, B. Beschoten, G. Gunterrodt, U. Nowak, and K. D. Usadel, Phys. Rev. Lett. 84, 4224 (2000).

1 L. L. Hinchey and D. L. Mills, Phys. Rev. B 34, 1689 (1986).

$1 \quad$ N. C. Koon, Phys. Rev. Lett. 78, 4865 (1997).

1 T. J. Moran and I. K. Schuller, J. Appl. Phys. 79, 5109 (1996); Y. Ijiri, J. A. Borchers, R. W. Erwin, S.-H. Lee, P. J. van der Zaag, and R. M. Wolf, Phys. Rev. Lett. 80, 608 (1998); T. J. Moran, J. Nogues, D. Lederman, I. K. Schuller, and K. V. Rao, Appl. Phys. Lett. 72, 617 (1998); J. A. Borchers, Y. Ijiri, D. M. Lind, P. G. Ivanov, R. W. Erwin, A. Qasba, S. H. Lee, K. V. O'Donovan, and D. C. Dender, Appl. Phys. Lett. 77, 4187 (2000).

1 T. C. Schulthess and W. H. Butler, Phys. Rev. Lett. 81, 4516 (1998); R. E. Camley, B. V. McGrath, R. J. Astalos, R. L. Stamps, Joo-Von Kim, and Leonard Wee, J. Vac. Sci. Technol. A 17, 1335 (1999); M. Kiwi, J. Mejia-Lopez, R. D. Portugal, and R. Ramirez, Europhys. Lett. 48, 573 (1999).

1 M. D. Stiles and R. D. McMichael, Phys. Rev. B 59, 3722 (1999); M. D. Stiles and R. D. McMichael, Phys. Rev. B 63, 4405 (2001); Z. F. Li and S. F. Zhang, Appl. Phys. Lett. 77, 423 (2000); Z. F. Li and S. F. Zhang, Phys. Rev. B 61, R14897 (2000); C. Leigton, J. Nogues, B. J. Jonsson-Akerman, I. K. Schuller, Phys. Rev. Lett 84, 3466 (2000). 
Appendix A

\title{
Wang-Landau Algorithm For Continuous Models and Joint Density of States
}

\author{
Cheggang Zhou \\ Computer Science and Mathematics Division,Oak Ridge National Laboratory \\ P.O. Box 2008, Oak Ridge Tennessee, 37831-6493 USA \\ and \\ Center for Simulational Physics, University of Georgia \\ Athens Georgia, 30602 USA \\ Thomas C. Schulthess \\ Computer Science and Mathematics Division, Oak Ridge National Laboratory \\ P.O. Box 2008, Oak Ridge, Tennessee, 37831-6493 USA \\ Stefan Torbrugge \\ Universit ät Osnabr ü ck, Fachbereich Physik \\ D-49076 Osnabr ü ck, Germany \\ David P. Landau \\ Center for Simulational Physics, University of Georgia \\ Athens Georgia, 30602 USA
}

\begin{abstract}
:
We present a modified Wang-Landau algorithm for models with continuous degrees of freedom. We demonstrate this algorithm with the calculation of the joint density of states of ferromagnet Heisenberg models and a model polymer chain. The joint density of states contains more information than the density of states of a single variable--energy, but is also much more time-consuming to calculate. We present strategies to significantly speed up this calculation for large systems over a large range of energy and order parameter.
\end{abstract}

PACS Numbers: 02.70.Tt, 02.70.Rr, 02.50.Fz, 02.50.Ey

The Wang-Landau (WL) algorithm [1] has been applied to a broad spectrum of interesting problems in statistical physics and biophysics $[1,2,3,9,5,4,7,8,6]$. These successful applications can be attributed to two features of this algorithm. First, the WL algorithm is not trapped by local energy minima. Secondly, by calculating the density of states, we can estimate thermodynamic observables including the free energy over a wide range of temperature with one single simulation. The efficiency and convergence of the WL algorithm has been quantitatively studied $[10,11]$, and variations $[7,8,6]$ and improvements have been proposed [4,5,12,13], among which the most interesting are those intended for systems with continuous degrees of freedom, e.g., protein [4] and 
liquids $[5,7,6]$. For such systems it is useful, but expensive, to calculate the joint density of states (JDOS) of two or more variables, e.g., $g(V, E)$ where $V$ is the volume and $E$ is the energy. JDOS is useful because quantities such as the free energy can be calculated as a function of temperature and pressure. Clearly, JDOS of continuous models provides information for many interesting problems, however the extra information does not come for free. Suppose we calculate the JDOS $g(M, E)$ of a $32 \times 32$ Ising model on a square lattice, a typical benchmark problem [1]. If every value of the magnetization $M$ is counted, $g(M, E)$ costs about $10^{3}$ times the CPU time of $g(E)$, because $g(M, E)$ contains about $10^{3}$ times as many entries as $g(E)$. Therefore such calculations have been restricted to small systems [14,5], or a carefully chosen small region in the parameter space [7,6]. Less costly alternative methods have been introduced, e.g., EXEDOS [4], which restricts the simulation at a fixed temperature instead of at a fixed external force field.

In this letter, we discuss two strategies to efficiently calculate JDOS for generic models with continuous degree of freedom, and give two examples. Our methods are equally applicable to systems with a phase transition, e.g., a classical Heisenberg ferromagnet, and biophysics systems, e.g., protein models. For the ferromagnet, we are interested in $g(M, E)$, where $M$ is the magnetization; while for protein models, we are interested in $g(\xi, E)$, where $\xi$ is a reaction coordinate [4].
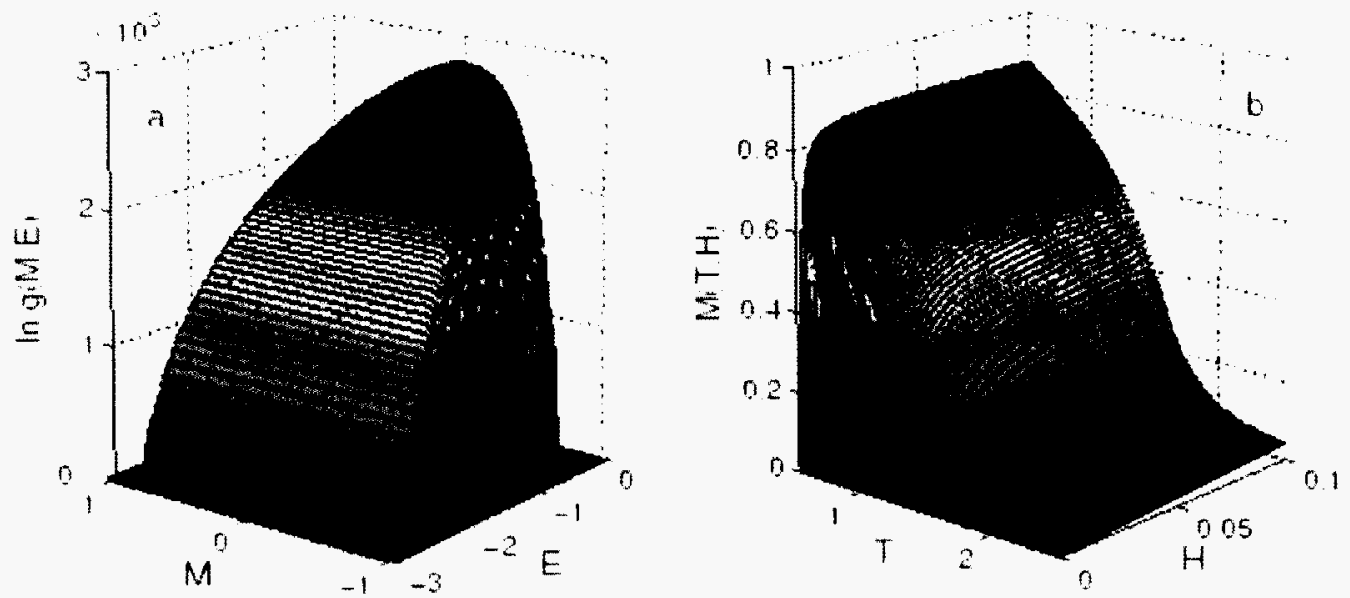

Figure 1: (color online) (a) $\ln g(M, E)$ of a three-dimensional Heisenberg ferromagnet of size $L=10$ with cutoff energy at -2.8 , determined up to an additive constant. (b) The magnetization at different temperature and external field evaluated from $g(M, E)$.

We first show the result of our calculation for a three-dimensional Heisenberg ferromagnet in Fig.1. The Hamiltonian of this model is $H=-\sum_{<i, j>} S_{i} \cdot S_{j}$, where the summation goes over nearest neighbors on a cubic lattice of size $L$ with periodic boundary conditions. This model has a ferromagnetic phase transition and displays global spin rotational symmetry. Here we define $E=H / L^{3}$ and $M=L^{-3} \sum_{i} S_{i}^{z}$. Figure 1(a) shows $\ln g(M, E)$ for negative $E$. A region with $\partial \ln g / \partial M=0$ below $E=-1.2$ indicates a transition to the ferromagnetic phase with a global rotational symmetry. Near 
the ground state $\ln g(M, E)$ is logarithmically divergent, since the JDOS vanishes when $E$ or $M$ is maximized or minimized. In the following, we use the Heisenberg ferromagnet as a prototype model to identify several features and intrinsic difficulties of continuous models.

In general, the models we study have many microscopic degrees of freedom $s=\left(s_{1}, \ldots, s_{N}\right)$, which labels the microscopic states in the phase space $\Omega$. The Hamiltonian $H(s)$ is a real-valued function of $s$, so is the order parameter, e.g., magnetization $M(s)$ for the Heisenberg ferromagnet. The JDOS is defined as

$$
g(M, E)=|\Omega|^{-1} \int \Pi_{i} d s_{i} \delta(E-H(s)) \delta(M-M(s)),
$$

where $|\Omega|$ is the volume of the phase space, and the integral is replaced by a summation for discrete models. We refer to a pair $(M, E)$ as a macroscopic state, and define the macroscopic phase space $\Lambda=\{(M, E) \mid \exists s, H(s)=E, M(s)=M\}$. Obviously $g(M, E) \geq 0$ is a probability measure of $\Lambda$ induced by an a priori probability measure of $\Omega$. In the following, we denote $(M, E)$ collectively with $x$. The WL algorithm learns $g(x)$ in with a simple strategy. In a word, it substitutes $\exp (-E / T)$ in a Metropolis algorithm with $\exp [-w(x)]$, where $w(x)$ is an approximation to $\ln g(x)$, and updates $w(x)$ with $w(x)+\ln f$ when the random walker arrives at state $x$. Here $f$ is called the modification factor. Previous studies [1,11] showed that for a discrete set of $x, w(x)$ quickly converges to $\ln g(x)$ within some statistical error proportional to $\sqrt{\gamma}$ for $\gamma \rightarrow 0$. For a continuous system, one cannot update $g(x)$ point by point but requires an algorithm that exploits the continuity of this function.

The simple binning scheme effectively approximates $g(x)$ with a piece-wise constant function. By using sufficiently many bins, this method does work for $g(E)[15]$; however, for JDOS this scheme results in an excessively large number of bins to sample, which will hamper the convergence. This can be avoided by using bilinear interpolation among neighboring bins [5]. Here we have adopted a kernel function update scheme similar to that of Ref. [16] for metadynamics, which seems more consistent with the continuous nature of $g(x)$. We select a localized positive continuous kernel function $k(x) \geq 0$, and scale it by two constants $\gamma$ and $\delta: k(x) \rightarrow \gamma k(x / \delta)$. If the random walker arrives in $x_{0}$, then the continuous histogram $w(x)$ is updated by

$$
w(x) \rightarrow w(x)+\gamma k\left(\left(x-x_{0}\right) / \delta\right),
$$

and we express the acceptance rate as

$$
A_{i \rightarrow f}=\min \left\{1, \exp \left[\ln \alpha \mid w\left(x_{i}\right)-w\left(x_{f}\right)\right] \mid\right\},
$$

where we have inserted a constant $\ln \alpha$ so that $w(x)$ converges to $\log _{\alpha} g(x)$. We have implemented a Gaussian kernel function $k(x)=\exp \left(-|x|^{2}\right)$, as well as an Epanechnikov kernel $k(x)=\left[1-|x|^{2}\right]_{+}$, and found no visible difference. By slightly modifying the approach in Ref. [11], we can prove the convergence of this scheme.

Thus the single modification factor in the original WL algorithm is replaced by a 
triplet $(\alpha, \gamma, \delta)$. In our simulations, we have used numbers between 0.0001 and 0.01 for $\gamma$, and $\delta$ corresponding to about $1 / 200$ of the width of the energy or magnetization windows. Unlike the original WL algorithm, we do not reduce $\gamma$ to extremely small values in the simulation, nor do we change $\delta$ in the simulation. We have the freedom to change $\alpha$ provided that $w(x)$ is properly rescaled.

If we start from an unbiased initial $w_{0}(x)=0$, we know $w_{T}(x)$ (where $T$ labels the number of Monte Carlo steps) grows from the region of large $g(x)$, and extends to unexplored region of small $g(x)$ [11]. $w_{T}(x)$ can be written as: $w_{T}(x)=\left[C(T)+\log _{\alpha} g(x)+r_{T}(x)\right]_{+}$, where $C(T)$ is an increasing function of $T$ only, $r_{T}(x)$ is a bounded error term controlled by the triplet $(\alpha, \gamma, \delta) . w_{T}(x)$ increases monoticically in the simulation and remains zero in the unexplored region. One cannot expect it to be flat as in the original WL algorithm for discrete models. The simulation should be stopped by other criteria, e.g., the visited area reaches a low energy cut-off, after which we continue the simulation with reduced $\gamma$ to improve the accuracy. If the result is accurate, $w_{T}(x)$ increases uniformly in the visited area. $T$ is estimated by counting the number of kernel functions to build up $w_{T}(x)$ :

$$
T=\left[\int \gamma k(x / \delta) d x\right]^{-1} \int_{\Lambda} w_{T}(x) d x .
$$

However, we find that the initial accumulation in which $\Lambda_{T}=\operatorname{supp}\left\{w_{T}(x)\right\}$ expands to $\Lambda$ takes a very long time for JDOS. The expansion of $\Lambda_{T}$ slows down as the area of $\Lambda_{T}$ increases. The reasons are two-fold. First, the simulation samples the macroscopic states within $\Lambda_{T}$ uniformly, giving rise to a uniform growth there. This uniform growth takes a considerable CPU time, while only about a fraction $\left|\Lambda_{T}\right|^{-1 / 2}$ of Monte Carlo steps on the boundary of $\Lambda_{T}$ happen to extend the simulation to the unexplored area. Secondly, close to the singular boundaries of $\Lambda, \nabla \log _{\alpha} g(x)$ becomes very large, requiring a very small $\delta$ (high resolution in the kernel function) to capture the large derivative.

To avoid repeated sampling of the visited region $\Lambda_{T}$, and to push the simulation to the unexplored region, we find it is most efficient to introduce a global update of $w_{T}(x)$ : when $w_{T}(x)$ is a good estimate of $\log _{\alpha} g(x)$ inside $\Lambda_{T}$, we update $w_{T}(x)$ with the following formula:

$$
w_{T}(x) \rightarrow w_{T}(x)+\kappa \exp \left[-\lambda w_{T}(x)-\omega\right] \Theta\left(w_{T}(x)-\omega\right),
$$

where $\Theta$ is the Heaviside step function. Basically, $w_{T}(x)$ is shifted up by an amount of $\kappa$ where $w_{T}(x)>\omega$, and the exponential function removes the resultant discontinuity. Here $\omega$ is positive because the local update scheme requires a minimum number of visits to give a correct estimation of the density of states. 

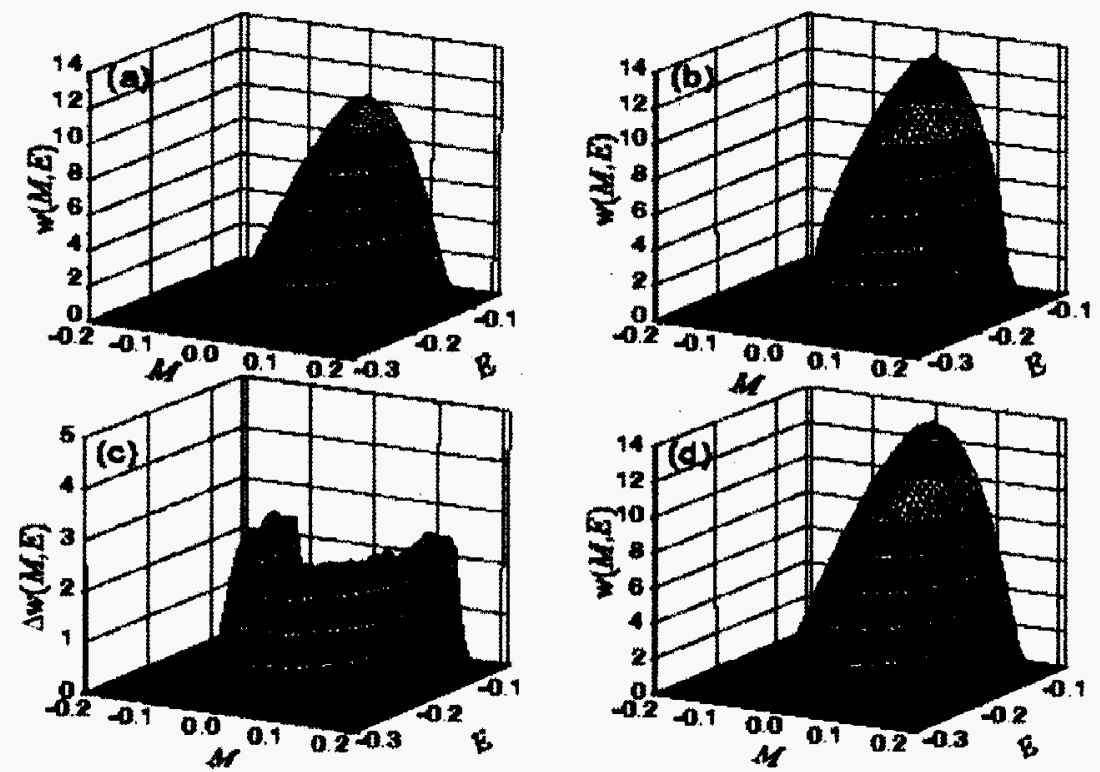

Figure 2: (color online) A cycle of the simulation with global update. (a) original $w_{T}(x)$, (b) $w_{T}(x)$ after the global update, (c) the increment accumulated beginning with the $w_{T}(x)$ in (b), (d) sum of (b) and (c) gives a new $w_{T}(x)$ in the end of this cycle.

After this global update, the random walker is forced to sample the boundary of $\Lambda_{T}$,

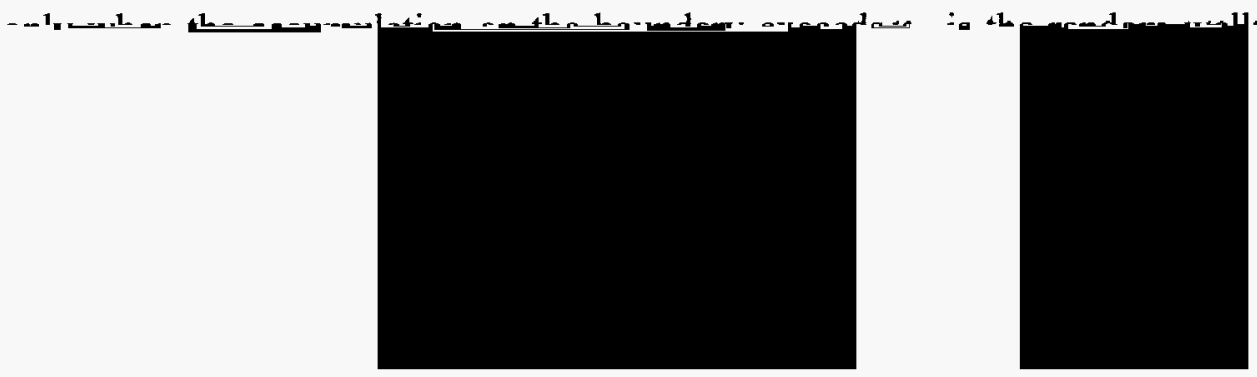


reduced. The short global samplings at the end of each cycle result in $T \propto W^{\lambda}$, with $\lambda \approx 1.55$ in Fig. 3 .

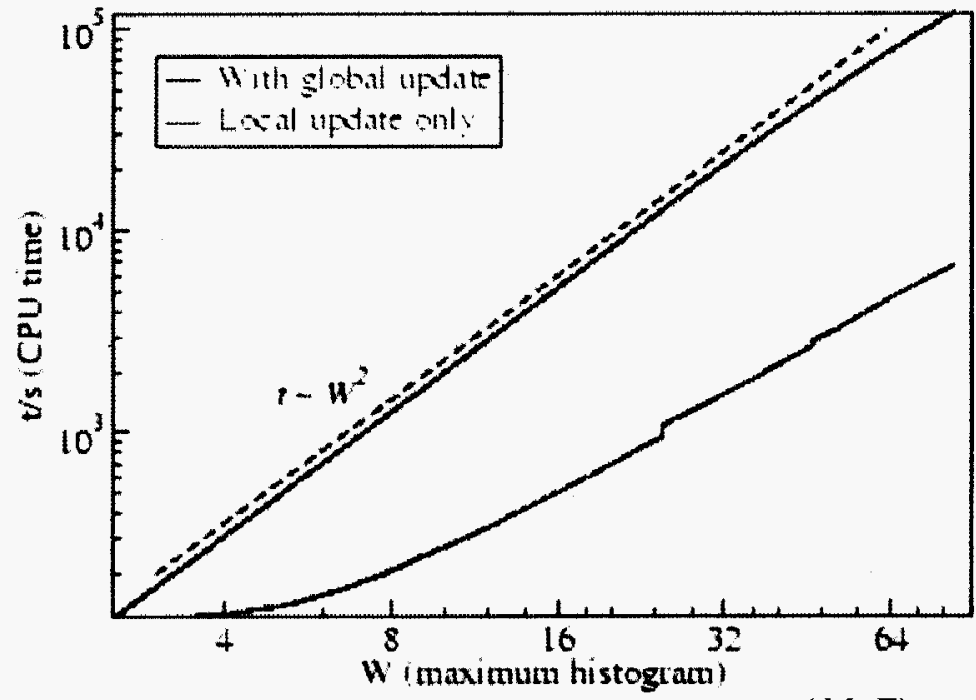

Figure 3: Comparison of CPU time used by the calculations of $g(M, E)$ with and without the global update for an $L=5$ Heisenberg model of ferromagnet, $\omega=0.5$ is used. The dashed line is a guide for the eye.

The algorithm can be summarized as the following: A calculation from scratch is divided into an initial accumulation stage and a refining stage. In the initial accumulation stage, (1) we start the calculation with $w(x)=0$, using local updates Eq. ((2)). (2) As soon as $w(x)>\omega$ for some $x$, we apply the global update Eq. ((5)), and continue the accumulation with local updates. $w_{T}(x)$ initially increases on the boundary of $\Lambda_{T} \cdot(3)$ After it resumes a uniform growth over $\Lambda_{T}$, we start the next cycle with another global update. The refining stage starts when $w_{T}(x)$ expands to the entire area of interest. Then we continue the simulation with only local updates until a uniform growth of $w_{T}(x)$ in this area observed. The JDOS can be refined with reduced $\gamma$ or taking average of multiple uncorrelated results of $w_{T}(x)$.

We calculate the thermodynamic quantities from $g(M, E)$ with numerical integral. Figure 1(b) shows the magnetization of Heisenberg ferromagnet as a function of external field and temperature. The specific heat in Fig. 4 shows a typical peak at $T_{c}$ of Heisenberg models. We also compare our results with that calculated with the original WL algorithm in Fig.4, which evaluates $g(E)$ on a grid of 3000 bins. They only differ slightly at low temperatures where both results show small errors. This error comes from the binning or interpolation scheme used to represent the continuous $g(E)$ or $g(M, E)$ (not from the numerical integration). 


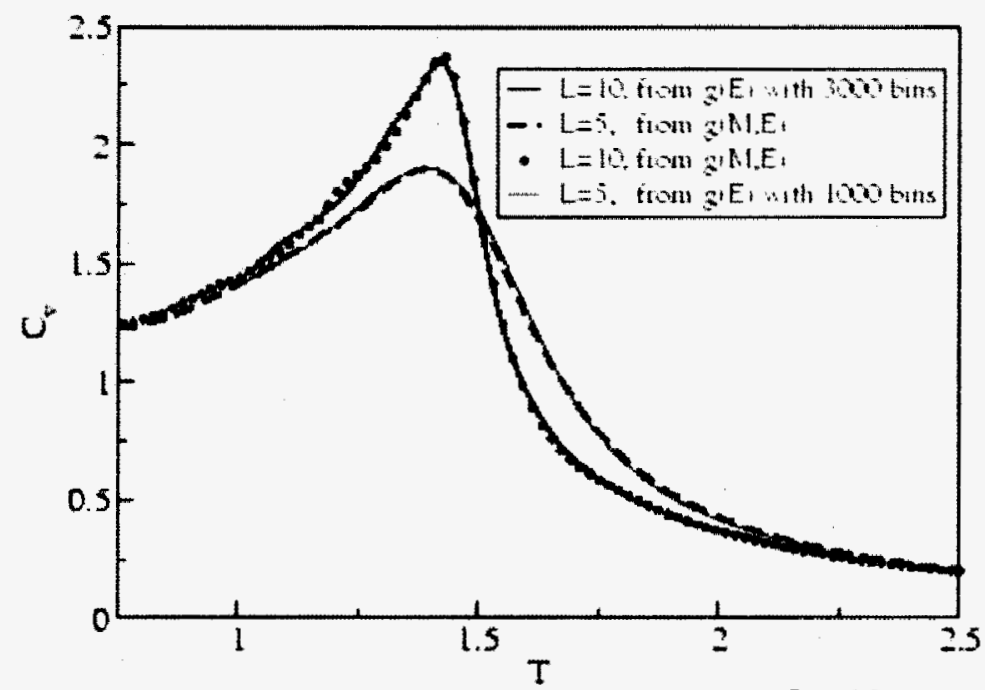

Figure 4: Specific heat of the Heisenberg ferromagnet of size $L=10$, and 5 , with comparison to the results from the original WL algorithm performed with a large number of bins.
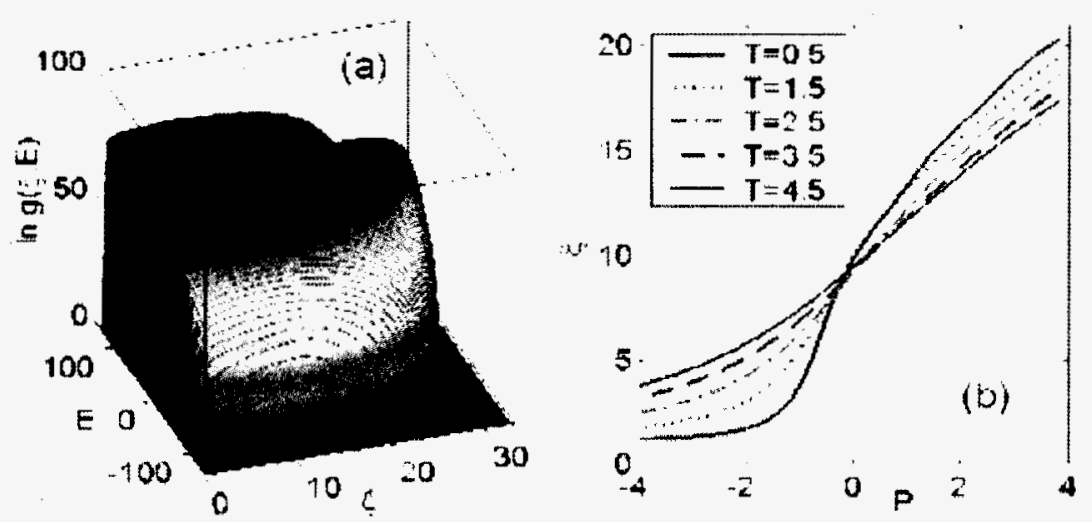

Figure 5: (color online)(a) JDOS $g(\xi, E)$ of a chain with 30 atoms. (b) The end-

to-end length $\xi$ as a function of pulling force $P$ at different temperatures.

Actually, given that the standard deviation of the canonical distribution of the energy is $\sigma_{E}=\sqrt{T^{2} C_{v} / L^{3}} \approx 0.036$ for $L=10, T=1$, and $\sigma_{E} \approx 0.1$ for $L=5$, an accurate numerical integration requires enough data points within $\sigma_{E}$. This criterion applies to both our kernel function updates and the bilinear interpolation scheme [5]. A larger $\sigma_{E}$ explains why the error is smaller for $L=5$ in Fig. 4 than that for $L=10$. In case of $L=10$, the internal array we used to store $g(M, E)$ has an energy resolution of 0.0012 , which is comparable to the bin size $(0.001)$ of the original WL algorithm we used for $g(E)$. Consequently, they show errors of comparable sizes. The conclusion is that the resolution in each macroscopic quantity must increase as $\sqrt{N}$ to maintain the accuracy in the numerical integral, where $N$ is the number of degrees of freedom.

Our second example is a simple protein model whose ground state is a perfect helix. The model is a polymer chain of fixed bond length and bond angle; its dihedral angles are 
the only degrees of freedom. We use our algorithm to calculate $g(\xi, E)$, where $\xi$ is the end-to-end distance (referred to as a reaction coordinate in Ref. [Pablo]) and we use the same parameters as in Ref. [Rapaport02] where it was originally studied. Figure 5 shows the JDOS and $\xi$ as a function of the pulling force $P$ at different temperatures for a chain of 30 atoms. A high energy cutoff is needed because the Lennard-Jones(LJ) repulsion has no upper bound. Due to the geometric constraints, the smallest polygon that the chain can form is a hexagon, in order to have a large LJ term. In this case, six bonds are replaced by the radius of the repulsion potential. Stretching the rest of the chain as much as possible, one can estimate that the threshold length is about $\xi=27$. This threshold gives rise to a clear shoulder structure in Fig $5(\mathrm{a}) . \xi(P, T)$ is calculated in the same way as $M(h, T)$ for the Heisenberg ferromagnet. At low $T$ and $P=0, \xi$ is the length of the helix, which behaves as a Hook spring for small pulling forces. The free energy for each $\xi$ (calculated with the EXEDOS algorithm in Ref. [4]) can be obtained by integrating $g(\xi, E) e^{-E / T}$ over $E$ directly. This example only took about half an hour on a single CPU.

In summary, we use kernel function local updates and a global update to extend the WL algorithm to efficiently treat continuous systems and their JDOS. Our new strategies have potential applications to many complex systems with thousands of degrees of freedom. In particular, the kernel function update benefits from the continuity of the model; and the global update effectively drives the random walker to unexplored areas, so that extreme values of macroscopic variables can be searched. Compared to the original WL algorithm, the global update saves about 90 percent CPU time in our calculations. Recently, we have also studied magnetic nanoparticles made of $\mathrm{NiFe}_{2} \mathrm{O}_{4}$ [18] with our method presented in this Letter.

We thank D. Sanders for his helpful comments. This research is supported by the Department of Energy through the Laboratory Technology Research Program and the Computational Materials Science Network, as well as the Division for Scientific User Facilities, and by NSF DMR-0341874.

\section{References}

[1] F. Wang and D. P. Landau, Phys. Rev. Lett. 86, 2050 (2001); Phys. Rev. E 64, 056101 (2001); Comput. Phys. Commun. 147, 674 (2002).

[2] C. Yamaguchi, and Y. Okabe, J. Phys. A 34, 8781 (2001).

[3] Y. Okabe, Y. Tomita, and C. Yamaguchi, Comput. Phys. Commun. 146, 63 (2002).

[4] N. Rathore and J. J. de Pablo, J. Chem. Phys. 116, 7225 (2002); N. Rathore, T. A. Knotts IV, and J. J. de Pablo, ibid. 118, 4285 (2003); N. Rathore, Q. Yan, and J. J. de Pablo, ibid. 120, 5781 (2004).

[5] M. S. Shell, P. G. Debenedetti, and A. Z. Panagiotopoulos, Phys. Rev. E 66, (056703 92002).

[6] E. A. Mastny and J. J. de Pablo, J. Chem. Phys. 122, 124109 (2005).

[7] Q. Yan, R. Faller, and J. J. de Pablo, J. Chem. Phys. 116, 8745 (2002); Q. Yan, and J. J. de Pablo, Phys. Rev. Lett. 90, 035701 (2003).

[8] S. Trebst, S. Trebst, D. A. Huse, and M. Troyer, Phys. Rev. E 70, 046701 (2004).

[9] M. Troyer, S. Wessel, and F. Alet, Phys. Rev. Lett. 90, 120201 (2003).

[10] P. Dayal, S. Trebst, S. Wessel, D. Wü rtz, M. Troyer, S. Sabhapandit, and 
S. N. Coppersmith, Phys. Rev. Lett. 92, 097201 (2004).

[11] C. Zhou and R. N. Bhatt, Phys. Rev. E 72, R025701 (2005).

[12] B. J. Schulz, K. Binder, M. Mü ller, and D. P. Landau, Phys. Rev. E 67, 067102 (2003).

[13] A. Malakis, A. Peratzakis, and N. G. Fytas, Phys. Rev. E 70, 066128 (2004).

[14] D. P. Landau, Shan-Ho Tsai, and M. Exler, Am. J. Phys. 72, 1294 (2004).

[15] G. Brown, and T. C. Schulthess, J. Appl. Phys. 97,10E303 (2005).

[16] C. Micheleti, A. Laio, and M. Parrinello, Phys. Rev. Lett. 92, 170601 (2004); Y. D. Wu, J. D. Schmitt, and R. Car, J. Chem. Phys. 121, 1193 (2004).

[17] D. C. Rapaport, Phys. Rev. E 66, 011906 (2002).

[18] C. Zhou, T. C. Schulthess, and D. P. Landau, cond-mat/0511410.

' T. C. Schulthess and W. H. Butler, "Magnetostatic Coupling in Spin Valves: Revisiting Nèel's Formula," Journal of Applied Physics, 87, 5759 (2000).

ii W. H. Butler, X.-G. Zhang, D. M. C. Nicholson, T. C. Schulthess, and J. M. MacLaren, "Giant Magnetoresistance from a Waveguide Effect in Cobalt-Copper Multilayers," Physical Review Letters, 76, $3216(1996)$.

iii D. J. Larson, P. H. Clifton, N. Tabat, A. Cerezo, A. K. Petford-Long, R. L. Martens and T. F. Kelly, "Atomic-scale analysis of CoFe/Cu and CoFe/NiFe interfaces", Applied Physics Letters, 77, 726 (2000).

iv M. K. Miller, A tom Probe Tomography, Kluwer Academic/ Plenum Publishers, New York (2000).

"For a review on the subject see: J. Nogues and I. K. Schuller, J. Magn. Magn. Mater. 192, 203 (1999); A. E. Berkowitz and K. Takano, J. Magn. Magn. Mater. 200, 552 (1999).

${ }^{v i}$ M. D. Stiles and R. D. McMichael, Phys. Rev. B 59, 3722 (1999); M. D. Stiles and R. D. McMichael, Phys. Rev. B 63, 4405 (2001); Z. F. Li and S. F. Zhang, Appl. Phys. Lett. 77, 423 (2000); Z. F. Li and S. F. Zhang, Phys. Rev. B 61, R14897 (2000); C. Leigton, J. Nogues, B. J. Jonsson-Akerman, I. K. Schuller, Phys. Rev. Lett 84, 3466 (2000).

vii J. S. Jiang, G. P. Felcher, A. Inomata, R. Goyetter, C. Nelson, and S. D. Bader, Phys. Rev. B 61, 9653 (2000).

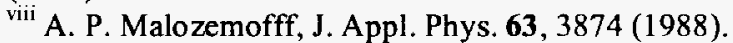

${ }^{i x}$ K. Takano, R. H. Kodama, A. E. Berkowitz, W. Cao, and G. Thomas, Phys. Rev. Lett. 79, 1130 (1997); P. Miltenyi, M. Gierlings, J. Keller, B. Beschoten, G. Gunterrodt, U. Nowak, and K. D. Usadel, Phys. Rev. Lett. 84, 4224 (2000).

${ }^{x}$ L. L. Hinchey and D. L. Mills, Phys. Rev. B 34, 1689 (1986).

${ }^{x i}$ N. C. Koon, Phys. Rev. Lett. 78, 4865 (1997).

xii T. J. Moran and I. K. Schuller, J. Appl. Phys. 79, 5109 (1996); Y. Ijiri, J. A. Borchers, R. W. Erwin, S.-H. Lee, P. J. van der Zaag, and R. M. Wolf, Phys. Rev. Lett. 80, 608 (1998); T. J. Moran, J. Nogues, D. Lederman, I. K. Schuller, and K. V. Rao, Appl. Phys. Lett. 72, 617 (1998); J. A. Borchers, Y. Ijiri, D. M. Lind, P. G. Ivanov, R. W. Erwin, A. Qasba, S. H. Lee, K. V. O'Donovan, and D. C. Dender, Appl. Phys. Lett. 77, 4187 (2000).

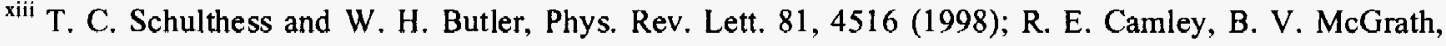
R. J. Astalos, R. L. Stamps, Joo-Von Kim, and Leonard Wee, J. Vac. Sci. Technol. A 17, 1335 (1999); M. Kiwi, J. Mejia-Lopez, R. D. Portugal, and R. Ramirez, Europhys. Lett. 48, 573 (1999).

${ }^{\text {xiv }}$ M. D. Stiles and R. D. McMichael, Phys. Rev. B 59, 3722 (1999); M. D. Stiles and R. D. McMichael, Phys. Rev. B 63, 4405 (2001); Z. F. Li and S. F. Zhang, Appl. Phys. Lett. 77, 423 (2000); Z. F. Li and S. F. Zhang, Phys. Rev. B 61, R14897 (2000); C. Leigton, J. Nogues, B. J. Jonsson-Akerman, I. K. Schuller, Phys. Rev. Lett 84, 3466 (2000). 